\title{
To Cryo-Cycle or Not to Cryo-Cycle; That is the Question!
}

Anchi Cheng ${ }^{1}$, William Rice ${ }^{1}$, Edward Eng ${ }^{1}$ and Michael Alink ${ }^{2}$

1. New York Structural Biology, Simons Electron Microscopy Center, National Resource for Automated Molecular Microscopy, New York, NY.

2. Thermo Fisher Scientific, Materials \& Structural Analysis, Hillsboro, OR.

There is currently very high demand for access to the highest end cryoEM instruments, such as the Titan Krios. Producing high-resolution reconstructions of protein complexes often requires capturing thousands of two-dimensional images. Any reduction in throughput capabilities adds delay to experimental timelines. There is thus pressure to maximize experimental throughput, which requires a careful analysis of the full microscope performance. Currently, the standard practices for microscope operation mode vary significantly at different sites. Each site has its own strategy which is aimed at optimizing the performance and the uptime of the instrument. One aspect of this strategy is the frequency and length of cryo-cycling of the instruments, i.e. a warmup cycle to expel water that has built up on the cold surfaces. This varies quite widely from site to site [1]; some sites cryo-cycle every week for 24 hours, others every other week for 48 hours, and numerous variants in between. Every hour of cryo-cycling adds significantly to tool unavailability. We thus set out to determine the optimal cryocycling strategy with the aim of minimizing instrument downtime, aimed at systems that have been installed for at least a year.

To achieve some understanding of the optimal cryo-cycle strategy we analyzed the water content inside the cryo box during a cryo-cycle using a residual gas analyzer (RGA). We found that water (molecular mass 18) partial pressure increases as expected at the start of the cryo-cycle but recovers within 12 hours. The water partial pressure was found to be lower than $\mathrm{H}_{2}$ partial pressure after cooling down to $\mathrm{LN}_{2}$ temperatures (see figure 1). Note that the RGA has a different sensitivity for different gases ( $\mathrm{H} 2$ is a leftover gas in most vacuum systems that has low pumping speeds). The graph in Figure 1 shows raw ion current through the RGA filter so this needs to be corrected for quantitative comparison (sensitivity for $\mathrm{H} 2$ being roughly half that of $\mathrm{H}_{2} \mathrm{O}$ ). We found that ice growth was essentially zero measured the day after a 12-hour cryo-cycle; the standard ice growth test showed intensity changes of about $0.03 \%$ (negligible) whereas soon after system install a value of $0.2 \%$ was measured.

We conclude that for a system that has been installed for at least one year, a 12-hour cryo-cycle every week or every other week is sufficient to achieve optimal performance of the system [2].

References:

[1] Alewijnse B. et al, J Struct Biol. 199(3) (2017), p. 225.

[2] The authors acknowledge funding from the Simons Foundation (349247), NYSTAR, and the NIH National Institute of General Medical Sciences (GM103310) with additional support from the Agouron Institute (F00316) and NIH S10 (OD019994-01). 
Figure 1. Comparison of $\mathrm{H} 2 \mathrm{O}$ to $\mathrm{H} 2$ partial pressure [a.u.] on a linear scale during a cryo-cycle. The red arrow points to the water peak and the green arrow to molecular hydrogen. The partial pressures were measured 30 minutes after the start of a cryo-cycle after 4 weeks of continuous operation of a Krios. The system has been in operation for 1 year.

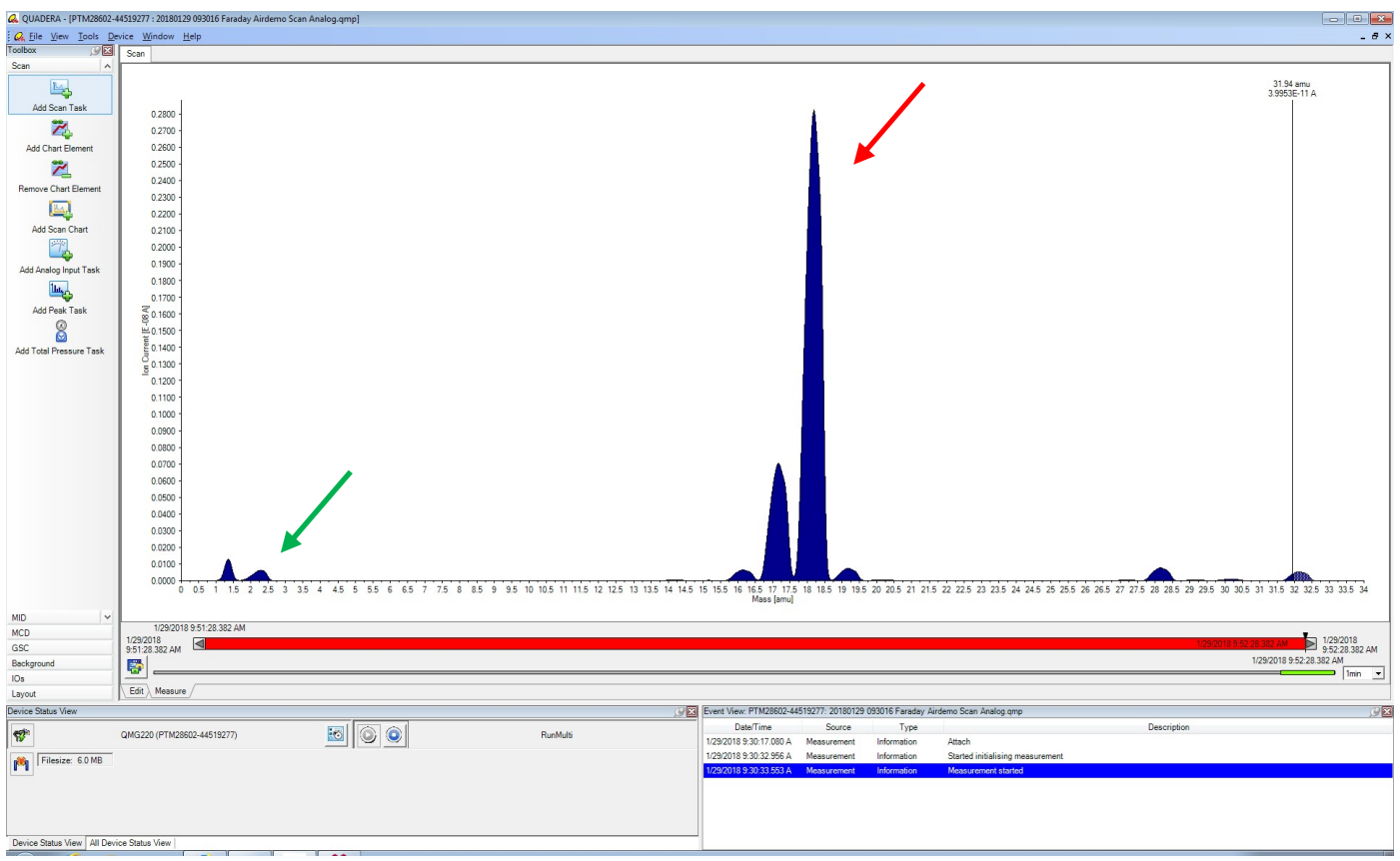

Figure 2. Comparison of $\mathrm{H} 2 \mathrm{O}$ to $\mathrm{H} 2$ partial pressure [a.u.] on a linear scale after 12-hour cryo-cycle and subsequent cooling of system to LN2. The red arrow points to the H2O peak and the green arrow to molecular hydrogen.

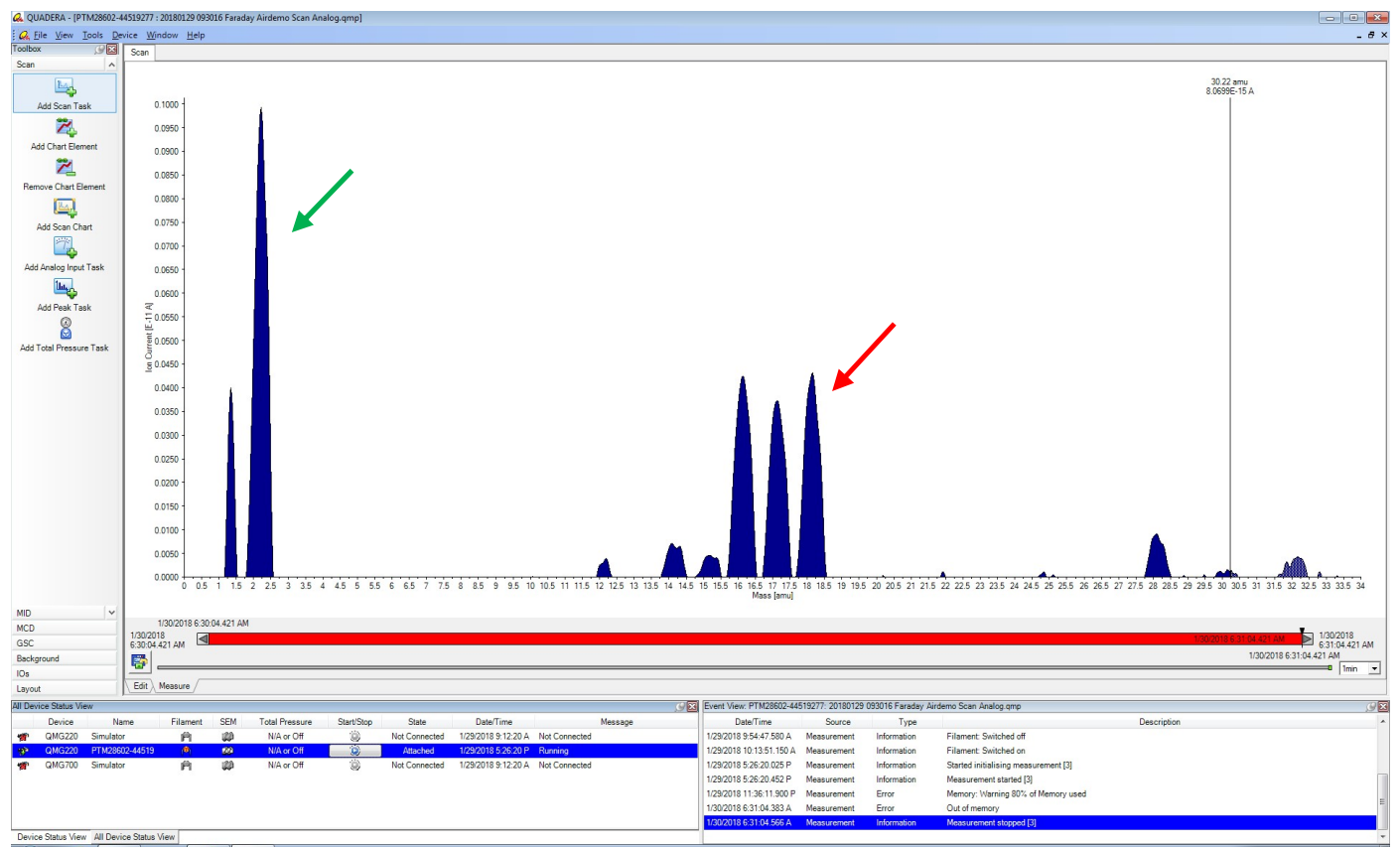

